ever, urine osmolality depends not only on secretion of antidiuretic hormone but also on the kidney's ability to respond. If the kidney's concentrating ability has been lost the urine will be dilute irrespective of the level of antidiuretic hormone, so the finding of a dilute urine in this situation does not by itself permit any conclusion to be drawn about antidiuretic hormone secretion. Furthermore, if the kidney is insensitive to antidiuretic hormone urine osmolality conveys no information on the setting of the osmostat, which can be assessed only by direct assay of antidiuretic hormone as a function of serum osmolality

\section{Totally implantable vascular access for long term chemotherapy}

Dr M F McMullin (Royal Victoria Hospital, Belfast BT12 6BA) writes: Following the comments of $\mathrm{Mr} \mathrm{A}$ E Young and colleagues ( 7 December, p 1608) I would like to draw attention to another advantage of the Port-a-Cath. In this unit two patients have used the Hickman catheter as a means of attempted suicide. Both patients, young men, had Hickman catheters inserted for intensive chemotherapy, and both attempted suicide by removing large quantities of blood through the catheter. Despite the problems of assessment, psychiatric disorders are common among patients with cancer, ${ }^{12}$ and cancer can increase the risk of suicide. ${ }^{3}$ In both these patients the Hickman catheter, which the patients manipulated daily themselves, was an easy means of access to the circulation. Perhaps the insertion of a Port-a-Cath would have avoided this complication.

1 Derogatis LR, Morrow GR, Fetting J, et al. The prevalence of psychiatric disorders among cancer patients. fAMA 1983, psychiatric

2 Maguire GP, Lee EG, Bevington DJ, Kuchemann CS, Crabtree RJ, Cornell CE. Psychiatric problems in the first year after mastectomy. Br Med J 1978;i:963-5.

3 Marshall JR, Burnett W, Brasure J. On precipitating factors: cancer as a cause of suicide. Suicide and Life-Threatening Behaviour 1983;13:15-27.

\section{Compression fractures of the dorsal spine in hypoglycaemic fits in diabetes}

Dr A J Richards (Southlands Hospital, Shorehamby-Sea, West Sussex BN4 6TQ) writes: I was interested to read the report by Sir John Nabarro on compression fractures of the dorsal spine after hypoglycaemic attacks in diabetes ( 9 November, p 1320). I have recently seen a similar case, a 52 year old insulin dependent diabetic who developed an episode of back pain after nocturnal hypoglycaemia. At no time was there any evidence of a convulsion, but recent radiographs show partial collapse of the dorsal vertebral bodies T5 to T8. Blood tests showed a raised alkaline phosphatase value but no other abnormality. He has had no further attacks and his back pain is subsiding, but, as Sir John states, back pain due to compression fractures after hypoglycaemic attacks in diabetics is probably more common than is generally realised.

\section{Psychiatric care for the mentally handicapped}

Dr T L Pilkington (Department of Psychiatry, University of Leeds, Leeds LS2 9LT) writes: The main point of my letter (26 October, $p$ 1201) was to draw attention to an issue of increasing concern in mental handicap practice: the problem of those who require various forms of psychiatric care and treatment. Whether this would be resolved simply by appointing more consultants as Dr D A Spencer implies (30 November, $p$ 1577) is arguable, as there is also the equally pressing need for the employment of more nurses with psychiatric training. Moreover, in March 1983 substantive appointments had not been made in 49 of the 150 funded consultant posts in England (Pilkington TL, paper at Seventh Congress of World Psychiatric Association, 1983) mainly because of the dearth of suitable applicants. Twenty three of these posts were occupied by locums. This survey is presently being updated but there is little evidence so far of any substantial improvement. Perhaps some rearrangement of the priorities in the existing services would have the most practical effect; certainly in many areas the present strategies do not appear to be treating the parts the others cannot reach.

\section{Banned mucolytic drugs}

Dr John Mason (Revlon Health Care Ltd, Berk Pharmaceuticals Ltd, Eastbourne, Sussex BN21 3YG) writes: Dr R A Keable-Elliott is not alone in questioning the decision and advice of the advisory committee on NHS drugs to the Minister for Health (23 November, p 1508) or in his sentiments.... As he points out, there is a real clinical need in some patients for oral mucolytics for which there are "no exact equivalents" available on NHS prescription. This should surely be the sole criterion for the availability of a medicine on National Health Service prescription. To allow children with tracheostomies the drug on NHS prescription is a laudable, humane gesture, which presumably incorporates also a recognition of clinical need but not necessarily proved efficacy. To deny the drug to patients who have had laryngectomies or who have serious chest conditions or "glue ear" on the grounds of unproved efficacy is therefore irrational and cruel.

\section{Communication from "centres of excellence"}

Professor J LORBER (Sheffield S11 9PN) writes: I was sorry to read Mr R J Brereton's letter (30 November, $p$ 1571) and the immense frustration he, his patients, and the doctors referring patients to him suffer as a result of inadequate secretarial help. I am replying in case people get the impression that this is the rule in hospitals. I have worked at the Children's Hospital in Sheffield, a "centre of excellence," for over 30 years. I invariably had adequate excellent secretarial help for both myself and my junior staff. We never had to wait a day before dictated letters were ready for signing and I never saw any excuse for anybody not sending reports to doctors within two days at most. Since I retired I have worked as a consultant locum in many non-teaching hospitals in the Trent Region. In every hospital I have been given a secretary. These secretaries were most competent and were prompt in typing and posting letters. This letter may be taken as an expression of my appreciation to the Trent Regional Health Authority and to the secretaries, who are invariably of the highest quality and most obliging. In general I do not agree with delays in sending letters out. I suspect that most delays occur because consultants or junior staff do not appreciate that their patients and family doctors need and deserve prompt and accurate information. I see no reason why they should not have it.

\section{Awards for research into crippling diseases}

Mr JoHn WaLFoRd (The Multiple Sclerosis Society, London SW6 lEE) writes: I was a little surprised to see the claim made that the grant of over $£ 358000$ made to Professor John Scales and his colleagues at the Institute of Orthopaedics was "the biggest single research award ever made by a charity" ( 30 November, p 1586). This is most certainly not so, this society having made a grant of well over $£$ lm to the National Hospital for Nervous Diseases for buying and maintaining a nuclear magnetic resonance scanner. I do not claim that this grant is the largest research award ever made by a charity; I would be surprised if it were.

\section{Safety of handling cytotoxic agents}

Dr R S Kingswell (Parke-Davis Research Laboratories, Eastleigh, Southampton) writes: Professor B M Colls ( 9 November, $p$ 1319) notes that many pharmaceutical companies neglect their responsibilities with respect to information on the handling and disposal of cytotoxic drugs. I should like to point out that amascrine, available in the UK and many other countries as Amsidine, carries with it a package insert which in addition to details on preparation of the infusion also provides information on what to do in the event of a spillage and on disposal of the preparation and contaminated materials. This information is given under "Pharmaceutical precautions," which also directs the reader to consult local hospital procedures and the Pharmaceutical Society's guidelines for handling of cytotoxic drugs. In its guidelines the Pharmaceutical Society suggests that cytotoxic agents should be prepared in an aseptic unit of a pharmacy with a vertical laminar flow cabinet, which should preferably be reserved solely for cytotoxic preparations. ${ }^{1}$

Working Party. Guidelines for the handling of cytotoxic drugs. Pharmaceutical fournal 1983; February 26:230-1.

\section{Isaac Newton}

Dr Milo Keynes (Department of Anatomy, University of Cambridge, Cambridge) writes: Dr Anthony Storr's discussion on Newton's psychopathology (2128 December, $p$ 1779) is finely done, but is it justified to set aside any real pathology by lack of emphasis? The purely psychological diagnoses that Dr Storr evokes must surely yield to consideration of the organic findings that came to light six years ago when raised concentrations of mercury in some of Newton's hairs were reported ${ }^{1}$; he mentions none of the subsequent literature. Newton was mentally ill during $1692-4$, and it has been suggested that his symptoms were due to mercury poisoning. Newton plainly exposed himself over 14 years before 1692 to the risk of metallic poisoning when he carried out several hundred alchemical experiments. Newton's mental disturbance consisted of melancholia, fears of persecution, sleeplessness, loss of appetite, and timidity, especially in the presence of strangers, or erethismus. Dr Storr points out that there was no mention of tremor or loss of teeth and that the symptoms subsided too quickly for the diagnosis of mercury poisoning to be likely. But tremor need not have been mentioned specifically, though Lettvin and Seitz, for instance, thought that a letter to Locke dated 2 August 1692 showed a tremulous handwriting. ${ }^{2}$ Mercurialism often shows stomatitis and gingivitis followed sometimes by loss of teeth but not necessarily. The suggestion of mercury poisoning, however unsatisfactory, cannot be lightly dismissed in a discussion of Newton's adult character, as done by Dr Storr. The possibility of such poisoning surely must be a likely explanation of a mental illness with features that largely correspond with that diagnosis.

1 Spargo PE, Pounds CA. Newton's 'derangement of the intellect': a new light on an old problem. Notes and Records of The Royal Sociery of London 1979;34:11-32.

2 Anonymous. Mercury, not mum? New Scientist 1971;52:274.

\section{Newton's law}

Dr Albert Ryan (Adlington, Macclesfield, Cheshire) writes: May I refer to Dr Anthony Storr's article on Isaac Newton (21-28 December, $p$ 1779) and point out that the law of gravitation states that everybody attracts every other with a force inversely (not directly as stated) proportional to the square of the distance between them. The law is not complete without also stating that the force is directly proportional to the product of their masses.

\section{Correction}

What is "serum albumin"?

We regret that an error occurred in this letter by Drs $P$ G Hill and J S Harrop (4 January, p 61). The first two sentences of the third paragraph shoud have read: "The figure compares the distribution of plasma albumin values in 1000 inpatients measured by the non-specific bromocresol green method and by a specific method using bromocresol purple. The accuracy of the bromocresol purple method compare favourably with that of immunochemical methods." 